\title{
Differentiation of mouse nuclear transfer embryonic stem cells into functional pancreatic beta cells
}

\author{
W. Jiang $\cdot$ Z. Bai $\cdot$ D. Zhang $\cdot$ Y. Shi $\cdot$ J. Yong $\cdot$ \\ S. Chen $\cdot$ M. Ding $\cdot$ H. Deng
}

Received: 13 January 2008 / Accepted: 18 April 2008 / Published online: 26 June 2008

(C) Springer-Verlag 2008

\begin{abstract}
Aims/hypothesis Therapeutic cloning has been reported to have potential in the treatment of several degenerative diseases. However, it has yet to be determined whether mouse nuclear transfer-embryonic stem cells (NT-ESCs) can be differentiated into pancreatic beta cells and used to reverse diabetes in an animal model.

Methods We first used the somatic nuclear transfer technique to generate mouse NT-ESCs and then developed a chemically defined stepwise protocol to direct the NT-ESCs into functional pancreatic beta cells. We examined the gene expression pattern of the differentiated NT-ESCs and transplanted the NT-ESCderived insulin-producing cells into recipient diabetic mice. Results Four mouse NT-ESC lines were first established using an improved nuclear transfer technique and insulin-
\end{abstract}

W. Jiang and Z. Bai contributed equally to this paper.

Electronic supplementary material The online version of this article (doi:10.1007/s00125-008-1065-1) contains supplementary material, which is available to authorised users.

W. Jiang $\cdot$ Z. Bai $\cdot$ D. Zhang $\cdot$ Y. Shi $\cdot$ J. Yong $\cdot$ S. Chen $\cdot$

M. Ding $\cdot H$. Deng $(\bowtie)$

Key Laboratory of Cell Proliferation and Differentiation

of the Ministry of Education, College of Life Sciences,

Peking University,

Beijing 100871, China

e-mail: hongkui_deng@pku.edu.cn

\section{Z. Bai}

National Research Institute for Family Planning,

Beijing, China

D. Zhang $\cdot$ J. Yong $\cdot$ H. Deng

Peking University Shenzhen Graduate School,

Shenzhen, China

Y. Shi

Chemistry Department, The Scripps Research Institute,

La Jolla, CA, USA producing cells were efficiently generated from NT-ESCs by mimicking pancreatic in vivo development. Most of the insulin-producing cells that we generated co-produced pancreatic and duodenal homeobox 1, but not glucagon at the final stage of this differentiation method, which differed from the insulin and glucagon co-production reported by other groups. The differentiated NT-ESCs were able to release insulin in response to glucose stimuli and normalise the blood glucose level of diabetic mice for at least 2 months.

Conclusions/interpretation These results demonstrate the potential of therapeutic cloning for cell therapy of type 1 diabetes in a mouse model.

Keywords NT-ESC · Nuclear transfer-embryonic stem cell · Pancreatic beta cell differentiation - Therapeutic cloning

$\begin{array}{ll}\text { Abbreviations } \\ \text { bFGF } & \text { basic fibroblast growth factor } \\ \text { EGF } & \text { epidermal growth factor } \\ \text { ESC } & \text { embryonic stem cell } \\ \text { FOXA2 } & \text { forkhead box A2 } \\ \text { ICM } & \text { inner cell mass } \\ \text { ITS } & \text { insulin-transferrin-selenium } \\ \text { NT } & \text { nuclear transfer } \\ \text { NT-ESC } & \text { nuclear transfer-embryonic stem cell } \\ \text { PDX1 } & \text { pancreatic and duodenal homeobox 1 } \\ \text { RA } & \text { all-trans retinoic acid } \\ \text { SOX } & \text { SRY (sex determining region Y)-box }\end{array}$

\section{Introduction}

Therapeutic cloning, which utilises healthy cells from a patient to create genetically matched embryonic stem cells (ESCs) by nuclear transfer (NT) and then drives the NT- 
ESCs to differentiate into specialised functional cells, offers an attractive potential in treatment of many degenerative diseases such as haemopoietic disease in mouse models [1]. However, it has not yet been reported whether NT-ESCs could be induced to differentiate into pancreatic beta cells and reverse diabetes in an animal model.

Type 1 diabetes results from loss of the pancreatic beta cells. At present, pancreatic islet transplantation has become a promising therapeutic option for patients with type 1 diabetes. However, this therapy is not widely used because of the severe shortage of donor islets and the problem of severe immune rejection. We hypothesised that a combination of the NT-ESC and differentiation techniques could be used to generate customised ESCs and to direct differentiation of the NT-ESCs into pancreatic beta cells as a promising resource for cell therapy to treat diabetes without requiring the use of immune-suppressor drugs.

Previously, ESCs have been shown to differentiate into pancreatic insulin-producing cells by our laboratory and other groups [2-6]. In our previous study, we established a stepwise induction method to differentiate mouse ESCs into functional pancreatic beta cells by treatment with a combination of activin A, all-trans retinoic acid (RA) and other maturation factors, and the differentiated pancreatic beta cells proved able to cure diabetic mice [3]. Recently, we adapted the protocol for human ESCs and successfully differentiated human ESCs into functional insulin-producing cells [5]. Several other groups have also developed protocols to differentiate human ESCs into insulin-producing cells in line with pancreatic development $[4,6]$. However, the reported efficiency is still very low in general, one of the major problems being that glucagon was co-produced with insulin in the differentiated cells $[4,6]$.

Here, we have successfully established mouse ESC lines by an improved NT technique and developed a chemically defined stepwise method to direct the NT-ESCs into functional pancreatic beta cells. Insulin-producing cells were effectively generated, which did not co-produce glucagon. The differentiated NT-ESCs expressed many pancreatic beta cell markers and secreted insulin in response to glucose and $\mathrm{KCl}$ stimulation. Furthermore, the NT-ESC-derived insulin-producing cells were transplanted into recipient diabetic mice and reversed hyperglycaemia.

\section{Methods}

Creation of cloned embryos The Institutional Animal Care and Use Committee of Peking University approved all animal procedures. Mouse fibroblast cells from the fetus F1 $(\mathrm{C} 57 \mathrm{BL} / 6 \times \mathrm{DBA})$ at 16.5 days post-coitum were cultured in DMEM (Gibco-BRL, Rockville, MD, USA) containing $20 \%$ FCS (vol./vol.; Gibco) until 100\% confluency and then exposed to DMEM supplemented with $0.5 \%$ FCS for 3 days for further NT as described previously [7]. The cloned embryos were cultured in KSOM medium (a potassium simplex optimised medium) [8] until the fourto eight-cell stage and subsequently treated by $0.05 \mu \mathrm{g} / \mathrm{ml}$ insulin for additional 3 days to the hatched blastocyst stage.

Establishment and characterisation of the NT-ESC lines For the establishment of the NT-ESC line, the cloned blastocysts were transferred into knockout DMEM (Gibco) with 20\% knockout serum replacement (vol./vol.; Gibco) containing 1,000 units/ml of leukemia inhibitory factor (Gibco) on a feeder layer treated with mitomycin-C (Roche, Indianapolis, IN). Following culture on the feeder for 13 to 14 days, the outgrowth was dissociated by needles and passaged for two times, then digested with trypsin to passage.

For teratoma assay, approximately $1 \times 10^{6} \mathrm{NT}$-ESCs were injected into the peritoneal of the donor mice and about 6 weeks later the injected mice were examined. The teratomas were embedded in paraffin and sectioned for histological analysis by haematoxylin-eosin staining. Additionally, a tetraploid blastocyst injection was performed to obtain cloned mice. About eight NT-ESCs were injected into the tetraploid blastocyst, assisted by a brief pulse of piezo micromanipulator, and the injected blastocysts were transferred into one uterine horn of pseudo-pregnant female mice at 2.5 days post coitum.

Differentiation of the NT-ESCs into pancreatic beta cells The protocol for differentiation of NT-ESCs into beta cells was modified and improved according to our previous reports $[3,5]$ and performed in a serum-free and chemically defined system. First, NT-ESCs were plated into Matrigelcoated (1:50; BD Biosciences, BD Biosciences, Bedford, MA, USA) Petri dishes (Nunc, Roskilde, Denmark) and incubated for 3 days with X-Vivo 10 (Cambrex, East Rutherford, NJ, USA) supplemented with $1 \mathrm{~g} / \mathrm{l}$ BSA (Sigma, St Louis, MO) containing $50 \mathrm{ng} / \mathrm{ml}$ activin A (Sigma). Then the differentiated cells were cultured for 2 days in F12/Iscove's modified Dulbecco's medium (1:1) supplemented with $5 \mathrm{~g} / 1 \mathrm{BSA}$ and $1 \%$ insulin-transferrinselenium (ITS, by vol.; all from Gibco) with $2 \mu \mathrm{mol} / \mathrm{l}$ RA (Sigma). After RA induction, the cells were cultured in DMEM (low glucose; Gibco) supplemented with $5 \mathrm{~g} / 1 \mathrm{BSA}$ and $1 \%$ ITS, together with $10 \mathrm{ng} / \mathrm{ml}$ basic fibroblast growth factor (bFGF; Sigma) and $20 \mathrm{ng} / \mathrm{ml}$ epidermal growth factor (EGF; Sigma) for 3 days and another 3 to 5 days in DF12 (Gibco) medium with ITS, $10 \mathrm{ng} / \mathrm{ml} \mathrm{bFGF}$ and $10 \mathrm{mmol} / \mathrm{l}$ nicotinamide (Sigma) for maturation.

Immunofluorescence assay The NT-ESCs or induced cells were fixed in $4 \%$ paraformaldehyde (wt/vol.) and blocked with $10 \%$ serum and $0.2 \%$ Triton X-100 (vol./vol.) in PBS, 
then incubated overnight at $4^{\circ} \mathrm{C}$ with primary antibody (Electronic supplementary material [ESM] Table 1) and further incubated with secondary antibody (fluorescein isothiocyanate (FITC) or tetramethylrhodamine B isothiocyanate (TRITC)-conjugated donkey anti-rabbit or anti-goat or anti-mouse IgG; Jackson Laboratories, West Grove, PA, USA). Images were captured using a phase contrast fluorescent microscope (IX-71; Olympus, Tokyo, Japan) or confocal microscope (TCS-SP2; Leica, Allendale, NJ, USA). Alkaline phosphatase activity was measured according to instructions of the manufacturer (Promega, Madison, WI, USA). The DeadEnd Fluorimetric TUNEL System (Promega) was used to perform the cell apoptosis assay.

The percentage of cells positive for pancreatic and duodenal homeobox 1 (PDX1) and C-peptide was calculated using Image-Pro Plus software (Media Cybernetics, Silver Spring, MD, USA) as follows: an area to be measured was outlined by a tracer, with the number of counting frames preset so that the device sampled the measuring sites randomly. The software counted the value of the respective chosen areas. We used DAPI staining to estimate the amount of whole cells. To count the percentage of cells positive for PDX1 or C-peptide, the positive areas were estimated by the software in the same way. The percentage was calculated by the following formula: area positive for C-peptide (or PDX1)/whole DAPI-positive area. Pictures were taken from at least three independent cultures and 50 random and different views of the differentiated cells to estimate the positive cell percentage.

RT-PCR analysis of gene expression Total RNA was extracted by TRIzol reagent (Invitrogen, Carlsbad, CA, USA). RNA was digested by RNase-free DNase I (Ambion, Austin, TX, USA) and then reverse-transcribed into cDNA by Moloney murine leukemia virus reverse transcriptase (Promega). PCR was performed with Ex Taq polymerase (TaKaRa, Otsu, Shiga, Japan) following the manufacturer's protocol. The cycle conditions were as follows: $94^{\circ} \mathrm{C}$ for $5 \mathrm{~min}$, followed by 35 cycles $\left(94^{\circ} \mathrm{C}\right.$ denaturation for $30 \mathrm{~s}$, $56-58^{\circ} \mathrm{C}$ annealing for $30 \mathrm{~s}, 72^{\circ} \mathrm{C}$ elongation for $35 \mathrm{~s}$ ), with a final incubation at $72^{\circ} \mathrm{C}$ for $6 \mathrm{~min}$.
Real-time PCR analysis was performed on a sequence detection system (ABI PRISM 7300; Applied Biosystem, Foster City, CA, USA) using the SYBR Green PCR Master Mix (Toyobo, Osaka, Japan). The PCR reaction consisted of $12.5 \mu \mathrm{l}$ of SYBR Green PCR Master Mix, $0.4 \mu \mathrm{mol} / \mathrm{l}$ of forward and reverse primers, $10.5 \mu \mathrm{l}$ water, and $1 \mu \mathrm{l}$ template cDNA in a total volume of $25 \mu 1$. The relative expression of each gene was normalised against Gapd (also known as Gapdh). The primers sequences and the length of the product are shown in ESM Table 2.

Insulin release assay by ELISA The insulin levels in culture supernatants were measured by a rat/mouse insulin ELISA kit (EZRMI-13K; Linco, St Charles, MO, USA). After preincubation with Krebs-Ringer buffer at $37^{\circ} \mathrm{C}$ for $90 \mathrm{~min}$, the differentiated cells were incubated at $37^{\circ} \mathrm{C}$ for $60 \mathrm{~min}$ with Krebs-Ringer buffer containing $2.5 \mathrm{mmol} / 1$ glucose or $27.5 \mathrm{mmol} / \mathrm{l}$ glucose or $30 \mathrm{mmol} / \mathrm{l} \mathrm{KCl}$. Then the respective conditioned mediums were collected and tested. The total protein content was detected with a protein assay kit (BCA; Pierce, Rockford, IL, USA).

Transplantation of insulin-producing cells under renal subcapsule Animals of the same strain of F1 male mice were injected with streptozotocin (Sigma; i.p., $180 \mathrm{mg} / \mathrm{kg}$ ) at about 8 weeks of age and used as experimental diabetes model. When the blood glucose level of streptozotocintreated mice was constantly above $13.9 \mathrm{mmol} / \mathrm{l}$, about $1 \times 10^{6}$ differentiated cells were transplanted into the left renal capsule. PBS-treated and the spontaneously differentiated NT-ESCs without factors treatment were used as control. Cryostat sections of the operated kidneys were prepared and C-peptide levels in transplanted cells in the renal capsule were tested by immunofluorescence.

\section{Results}

Establishment and characterisation of NT-ESC lines We synchronised the donor cells by combined contact inhibition and serum starvation treatment and obtained a high ratio of

Table 1 Effect of contact inhibition and/or serum starvation on in vitro development of mouse cloned embryos

\begin{tabular}{lllll}
\hline Parameter & Oocytes $(n)$ & Fused, $n(\%)$ & Cleaved, $n(\%)$ & Blastocysts, $n(\%)$ \\
\hline Contact inhibition & 319 & $281(88.1)$ & $135(48.0)^{\mathrm{a}}$ & $49(36.3)$ \\
Serum starvation & 267 & $232(86.9)$ & $141(60.8)^{\mathrm{b}}$ & $58(41.1)$ \\
Contact inhibition + serum starvation & 395 & $340(86.1)$ & $236(69.4)$ & $95(40.3)$ \\
\hline
\end{tabular}

Following combined contact inhibition and serum starvation treatment, the cleavage rate of the cloned embryos was significantly higher than that following serum starvation $(p<0.05)$ or contact inhibition treatment $(p<0.01)$, respectively. However, the blastocyst formation rate from the twocell stage did not differ among the three treatment groups

${ }^{\mathrm{a}} p<0.01$ vs serum starvation group (cleaved); ${ }^{\mathrm{b}} p<0.005$ vs contact inhibition + serum starvation (cleaved) 
Table 2 Effect of insulin on the in vitro development of cloned embryos

\begin{tabular}{llccccc}
\hline Medium & Fused oocytes $(n)$ & Cleaved, $n(\%)$ & Blastocysts, $n(\%)$ & Blastocysts tested, $n$ & Total cell number & ICM cell number \\
\hline K-KII & 92 & $57(62.0)$ & $28(49.1)$ & 15 & $63.2 \pm 15.6^{\mathrm{a}}$ & $17.1 \pm 5.7^{\mathrm{a}}$ \\
K-KII + insulin & 103 & $67(65.0)$ & $35(52.2)$ & 22 & $89.0 \pm 13.1$ & $28.0 \pm 7.0$ \\
\hline
\end{tabular}

Values are means \pm SEM, unless otherwise stated

When cloned embryos were transferred into the KSOM-II medium supplemented with $0.05 \mu \mathrm{g} / \mathrm{ml}$ insulin at the four to eight cell stage and cultured to blastocyst stage, the cell number within the cloned ICM was significantly enhanced $(p<0.05)$ compared with that of the control group, although the blastocyst formation rate $(p>0.05)$ did not improve

${ }^{\mathrm{a}} p<0.05$ for difference vs other value for K-KII + insulin in same column

K, KSOM; KII, KSOM supplemented with $0.5 \%$ essential amino acids and $0.5 \%$ non-essential amino acids (vol./vol.), and with concentration of glucose increased to $5.56 \mathrm{mmol} / 1$

cloned cleavage embryos (Table 1). Then the cloned four to eight cell stage embryos were transferred into the medium supplemented with $0.05 \mu \mathrm{g} / \mathrm{ml}$ insulin to significantly enhance the cell number within the cloned inner cell mass (ICM) of the blastocyst (Table 2). Following nine cloned blastocysts transferred into the ESC media, four ESC lines (44.4\%) were established. The resulting clone-derived ESCs had a high ratio of nucleus to cytoplasm as well as prominent nucleoli, and their colony morphology was similar to that of typical mouse ESCs (Fig. 1a).

The mouse NT-ESCs expressed specific pluripotent markers, including POU domain, class 5, transcription factor (POU5F1), Nanog homeobox (NANOG), SRY-box containing gene 2 (SOX2), stage-specific embryonic antigen 1 (SSEA1) and alkaline phosphatase (Fig. 1b-f, respectively). The ESCs had a normal karyotype (normal fraction $>80 \%$ ) (ESM Fig. 1a). Teratomas formed (ESM Fig. 1b) 6 weeks after the NT-ESCs injection and histological examination revealed that all three germ layers were generated: endoderm-derived gut epithelium (ESM Fig. 1c), ectoderm-derived neurons (ESM Fig. 1d), and
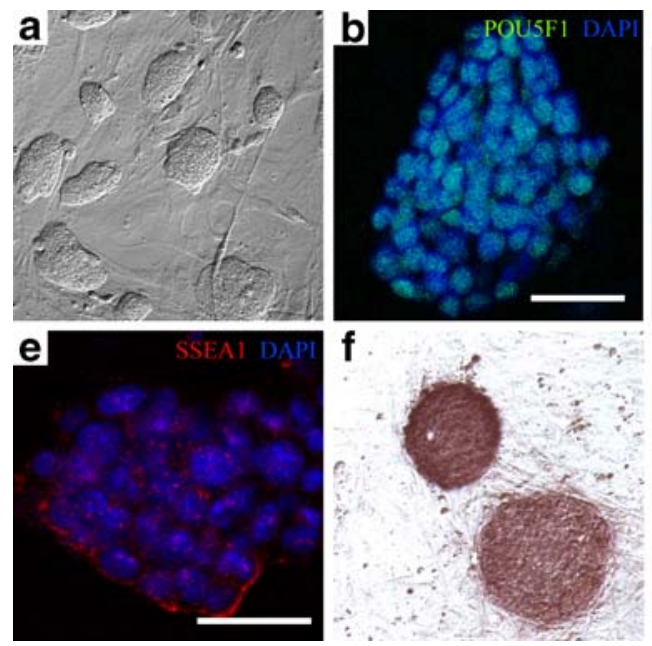

Fig. 1 Characterisation of the NT-ESCs. a Phase contrast of NT-ESCs growing on top of feeder layer. b-e Presence of POU5F1 (b), NANOG (c), SOX2 (d) and SSEA1 (e); scale bar, $40 \mu \mathrm{m}$. f Alkaline phosphatase staining of NT-ESCs. g Adult NT-ESC-tetraploid mice mesoderm-derived cartilage (ESM Fig. 1e) and adipose tissue (ESM Fig. 1f). Furthermore, four NT-ESC-tetraploid embryos developed into adults by tetraploid blastocyst injection (Fig. 1g) and delivered normal offspring (Fig. 1h) after natural mating.

Differentiation of NT-ESCs into beta cells in vitro We developed an efficient chemically defined protocol to differentiate the mouse NT-ESCs into functional pancreatic beta cells (Fig. 2a). Many T-positive cells were observed after 2 days with activin A treatment (Fig. 2b) and SOX17- and forkhead box A2 (FOXA2)-positive cells were also observed after 3 days with activin A treatment (Fig. 2c), suggesting that the NT-ESCs differentiated into mesendoderm then further into definitive endoderm cells. After RA treatment, PDX1-positive cells appeared (Fig. 2d). In the latter stage, the differentiated cells produced pancreatic endocrinal markers, including NEUROG3, NKX6-1, SOX9, PAX6 and ISL1 (Fig. 2e). These data suggested that the differentiation progress mimicked the in vivo pancreatic development to a certain extent.

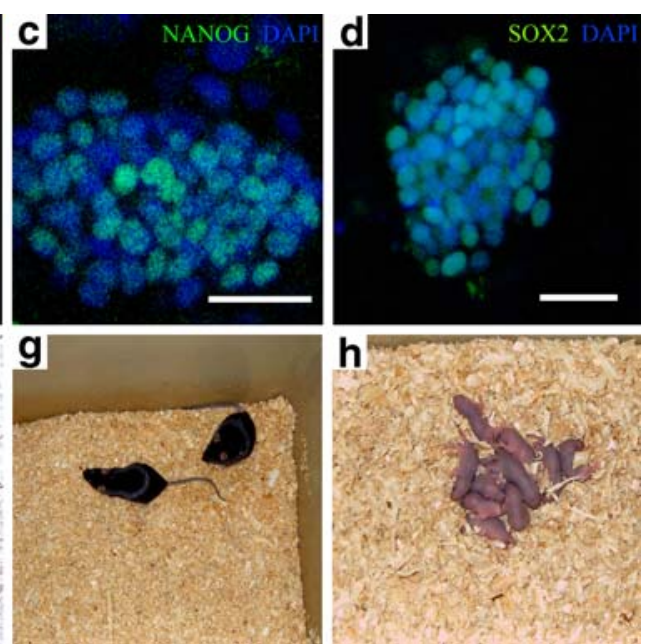

derived from NT-ESCs complemented by tetraploid embryos and h living offspring generated from the adult NT-ESC-tetraploid mice following natural mating 
In the final stage, visible clusters formed that were C-peptide-positive and PDX1-positive as examined by immunofluorescence (Fig. 3a). Many NKX6-1- and KCNJ11 (potassium inwardly-rectifying channel, subfamily $\mathrm{J}$, member 11)-positive cells were also observed (Fig. 4a,b). RT-PCR data showed that the differentiated cells expressed pancreatic beta cell-specific genes, including insulin I, insulin II, $P d x 1$, Iapp, Isll, $A b c c 8$ and $N k x 2-2$, and other endocrinal markers, including somatostatin and glucagon (Fig. 3c). We observed about 5 to $10 \%$ PDX1- and C-peptide-positive cells in the final stage (data not show). To further confirm that the clusters contained mature beta cells, we performed costaining of the C-peptide with PDX1 or glucagon. We found that most of the clusters of C-peptide-positive cells were PDX1-positive (Fig. 3a), but glucagon-negative (Fig. 3b). These data are consistent with the known gene expression pattern of mature islet cells.

To determine whether insulin secretion from those differentiated NT-ESCs could be regulated by glucose or other physiological stimuli, we treated these cells with a high concentration of glucose or $\mathrm{KCl}$ and then analysed the insulin release level in culture medium by ELISA. We found that the insulin release with high glucose stimulation was significantly higher than that with low glucose $(1.15 \pm 0.26$ vs $0.53 \pm 0.04 \mathrm{pmol} / \mathrm{mg}$ protein, $p=0.005$ ) and that the differentiated cells could also respond to $\mathrm{KCl}$ stimulation
Fig. 2 Characterisation of the differentiation process. a Flow chart of the differentiation protocol. b t-complex (T) produced at day 2; c SRY-box containing gene 17 (SOX17) and FOXA2 produced at day 3 ; d PDX1 produced at day 5; and e neurogenin 3 (NEUROG3), NK6 transcription factor related, locus 1 (Drosophila) (NKX6-1), SRY-box containing gene 9 (SOX9), paired box gene 6 (PAX6) and ISL1 transcription factor, LIM/homeodomain (ISL1) produced at day 8 . IMDM, Iscove's modified Dulbecco's medium. Scale bar, $100 \mu \mathrm{m} T$ brachury homologue a

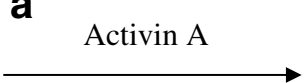

Ex vivo, 3 days

b

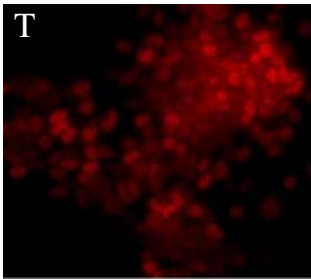

c
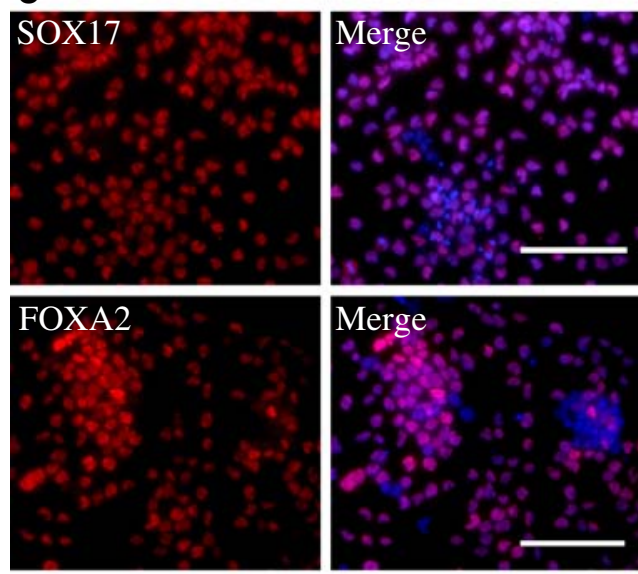

Merge

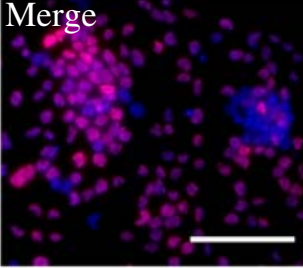

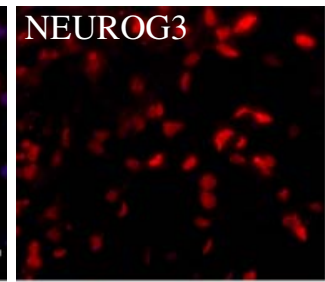

NKX6-1

d

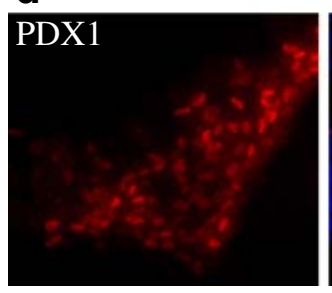

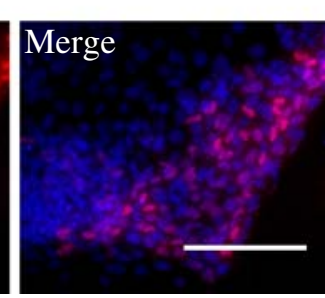

e
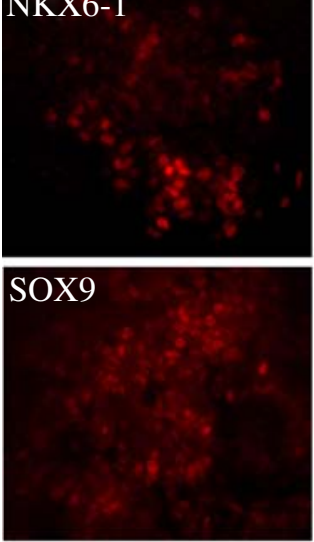

PAX6

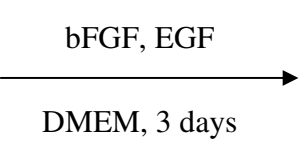

Nicotinamide, bFGF

DF12, 3-5 days
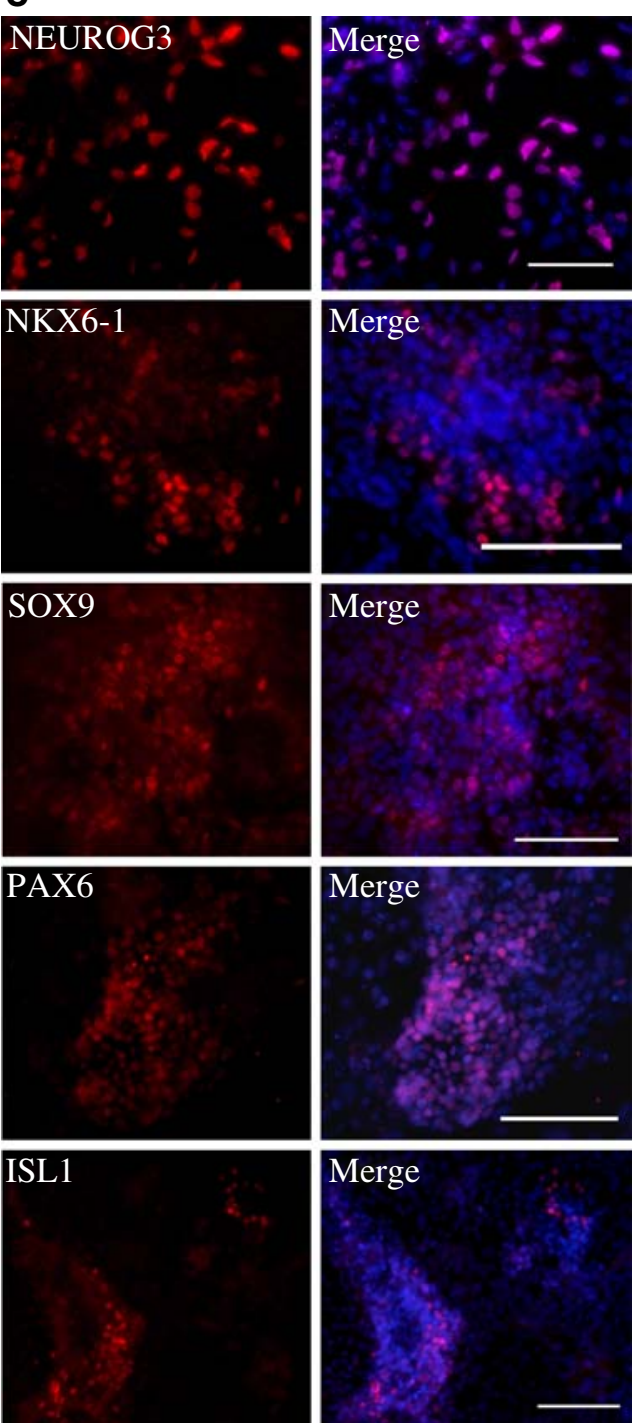

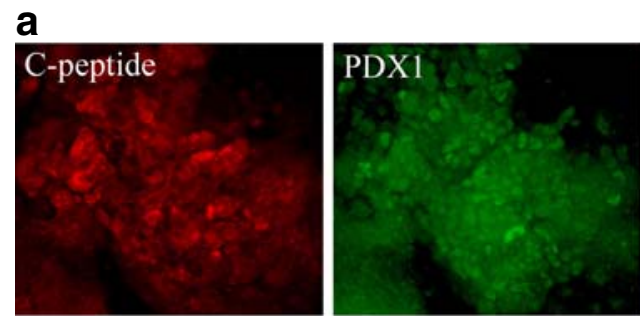

b

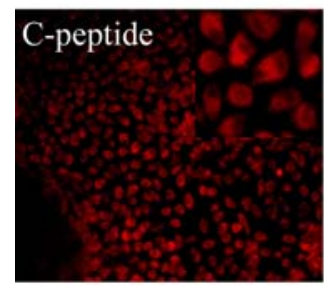

C

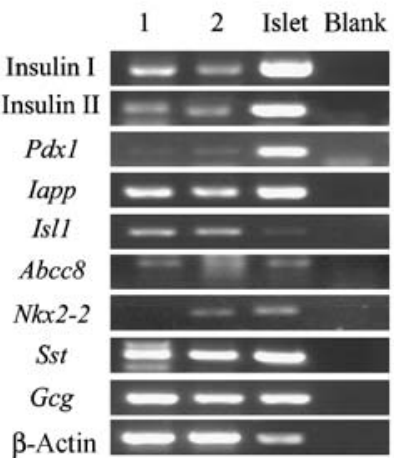

e
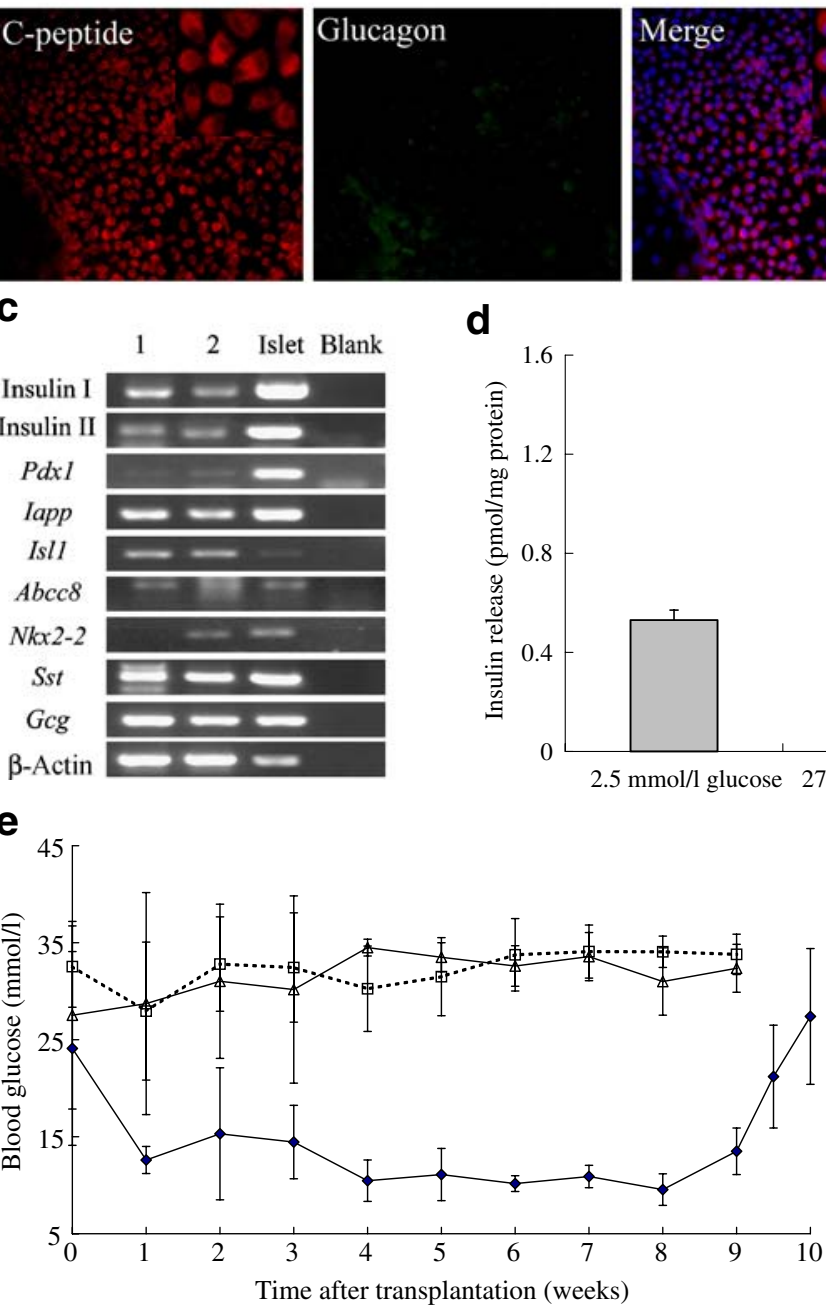

d
Positive control
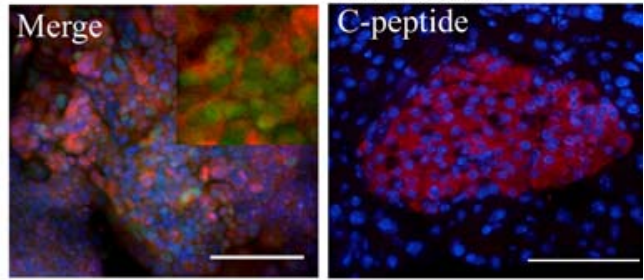

Positive control
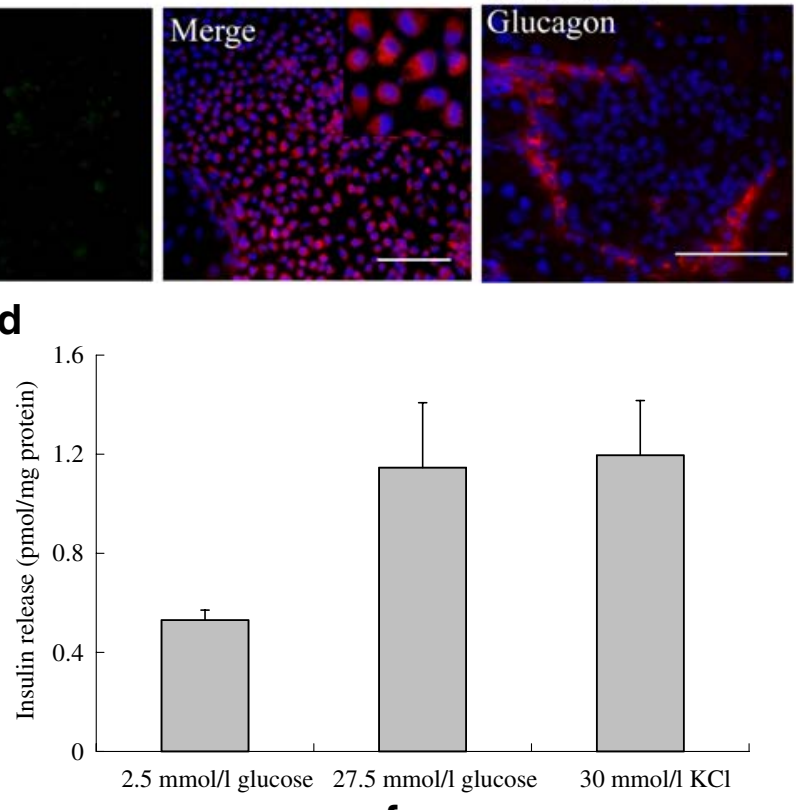

f

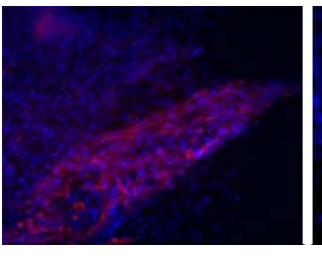

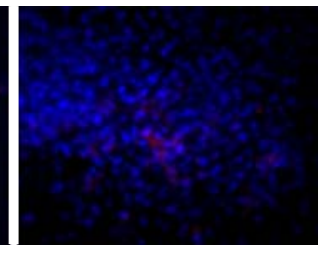

Fig. 3 Differentiation of NT-ESCs to functional pancreatic cells. a C-peptide- and PDX1-positive clusters derived from NT-ESCs, with positive control for C-peptide from cryostat section of mouse pancreas, as indicated. b Distribution of C-peptide and glucagon at the final stage, with positive control for glucagon from cryostat section of mouse pancreas, as indicated. Scale bar (a, b), $100 \mu \mathrm{m}$. c RT-PCR data of the differentiated NT-ESCs at the final stage. Lanes 1 and 2 represent two different experimental groups. Mouse islets were used as the positive

$(1.20 \pm 0.22$ vs $0.53 \pm 0.04 \mathrm{pmol} / \mathrm{mg}$ protein, $p=0.001)$. These data therefore indicated that the differentiated NTESCs secreted insulin in response to glucose and $\mathrm{KCl}$ stimulation (Fig. 3d). Considering that the medium contained insulin and apoptotic cells could take up the insulin, we performed the TUNEL assay and observed that controls. d Analysis of insulin release of induced cells treated with high and low concentration of glucose and $\mathrm{KCl} . p=0.005$ by Student's $t$ test for $2.5 \mathrm{mmol} / 1$ vs $27.5 \mathrm{mmol} / 1 ; p=0.001$ by Student's $t$ test for $2.5 \mathrm{mmol} / \mathrm{l}$ vs $30 \mathrm{mmol} / \mathrm{l}$. e The blood glucose of operated mice at different time points. Black diamonds, induced cells; white squares, PBS; white triangles, control cells (differentiated without factors treatment) f C-peptide staining in the grafted kidney of induced celltransplanted mice (left) and non-induced cell-transplanted mice (right)

PDX1-positive cells and C-peptide-positive cells were TUNEL-negative (Fig. 4c).

Transplantation of NT-ESC-derived insulin-producing cells reversed diabetes in diabetic mice After transplantation of NT-ESC-derived insulin-producing cells, the blood glucose of 
Fig. 4 Characterisation of the differentiation cell at the final stage. NK6 transcription factor related, locus 1 (Drosophila; NKX6-1) (a) and potassium inwardly rectifying channel, subfamily J, member 11 (KCNJ11) (b) were stained at the final differentiated NT-ESCderived cells. c The co-stain of TUNEL and PDX1 or C-peptide of the final differentiated NT-ESC-derived cells, with positive control of TUNEL by DNase treatment as indicated. d The co-stain of beta-III tubulin and C-peptide of the final differentiated NT-ESC-derived cells, with positive control of beta-III tubulin, as indicated. Scale bar, $100 \mu \mathrm{m}$
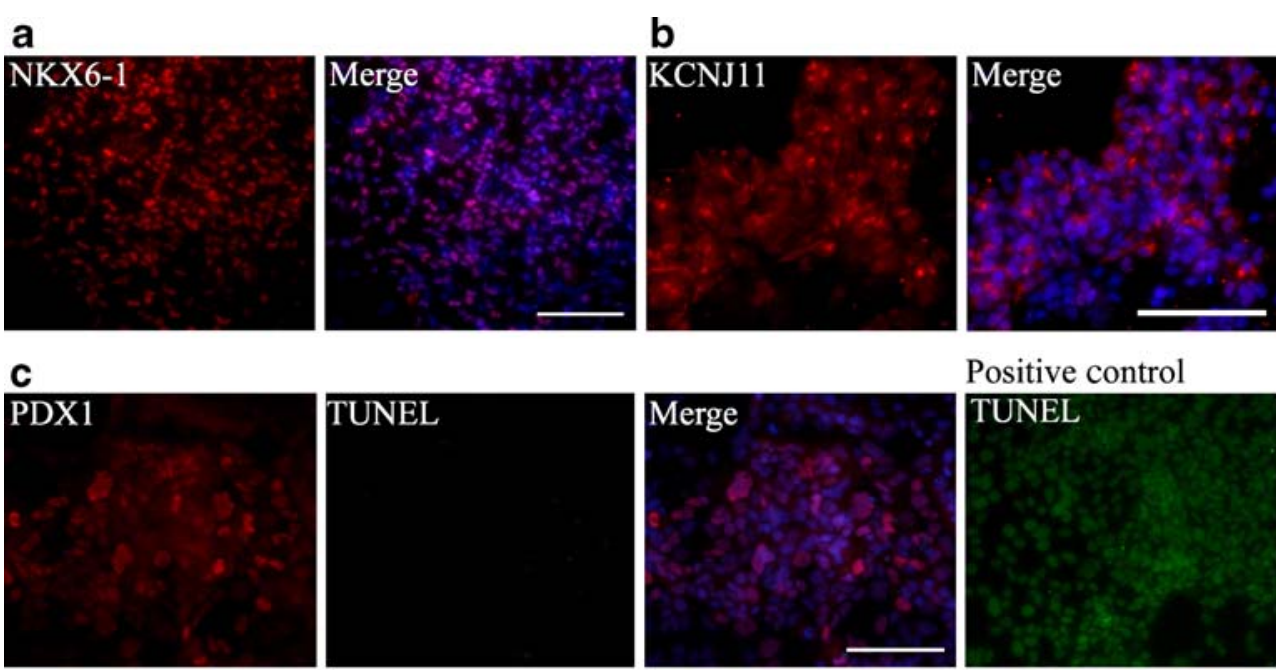

Positive control
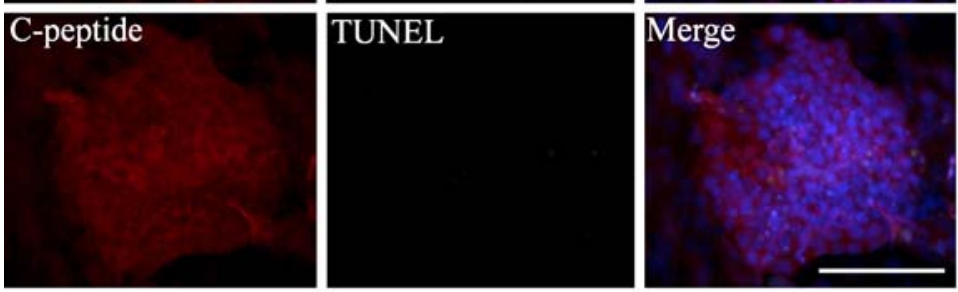

\section{TUNEL}
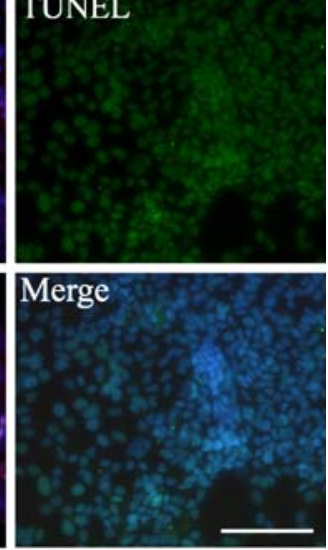

d
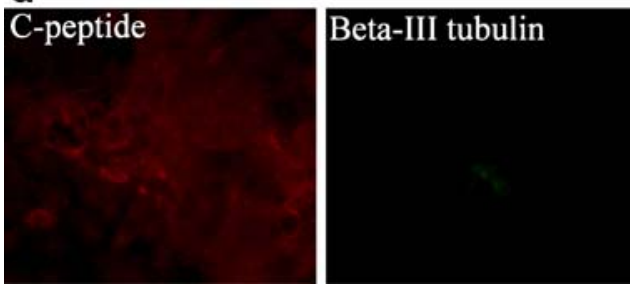

Positive control Beta-III tubulin

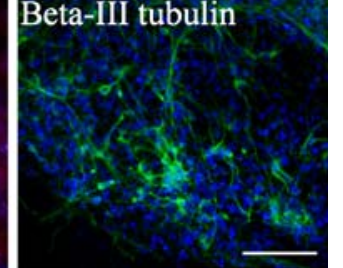

cell-treated mice $(n=5)$ was significantly reduced. In contrast, the blood glucose of the sham operated control (PBStransplanted) mice $(n=7)$ and the control cell-transplanted (without factors treatment group) mice $(n=4)$ remained above $20 \mathrm{mmol} / \mathrm{l}$. In addition, after removal of the left kidney transplanted with NT-ESC-derived beta cells, the blood glucose level reverted back to levels over $13.9 \mathrm{mmol} / \mathrm{l}$ (Fig. 3e).

C-peptide-positive cells were visualised in the cryostat sections of NT-ESC-derived beta cell-treated kidneys but not in the control cell-transplanted kidneys (Fig. 3f). These results indicate that the NT-ESC-derived insulin-producing cells improved glucose regulation and reversed the streptozotocin-induced diabetes in mice when transplanted into the renal capsule.

\section{Discussion}

The present study demonstrated the potential of therapeutic cloning in type 1 diabetes cell therapy. We successfully established four mouse ESC lines by modified NT methods and differentiated the NT-ESCs into functional insulinproducing cells that were able to reverse streptozotocininduced diabetes in mice.

A new and efficient chemically defined stepwise protocol for the differentiation of mouse NT-ESCs into insulin-producing cells was designed. Previously, we had developed a novel three-step protocol combined with activin $\mathrm{A}$ and RA to induce mouse ESC differentiation into pancreatic beta cells [3]. This was the first study demonstrating that the combination of activin A and RA could direct pancreatic islet fate from ESCs. The previous protocol was then successfully adapted for human ESC differentiation in a chemically defined system [5]. However, our previously established mouse protocol [3] involved spontaneous embryonic body formation and used fetal bovine serum, which could interfere with differentiation efficiency and reproducibility. In the present work, we developed a chemically defined protocol for cloned mouse ESCs that does not use embryonic body system. Furthermore, we found that EGF treatment could improve the 

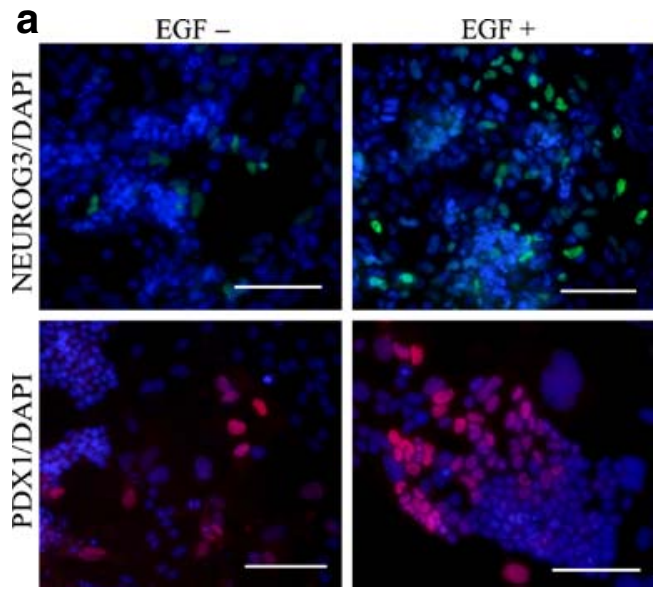

b

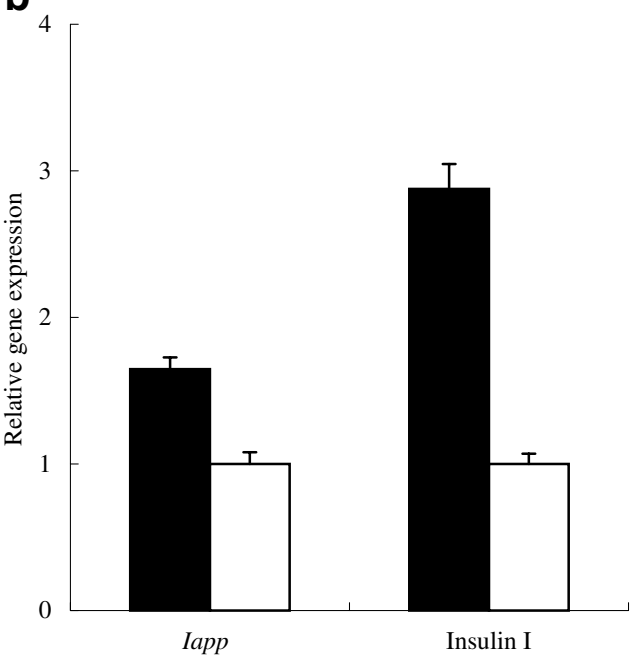

Fig. 5 Analysis of the effect of EGF treatment. a Immunostaining indicated that the neurogenin 3 (NEUROG3)-positive cells (at day 8) and PDX1-positive cells (at day 11) were significantly increased after EGF treatment. Scale bar, $100 \mu \mathrm{m}$. b The related Iapp and insulin I mRNA expression at the final stage after EGF treatment. The gene expression of groups without EGF treatment was normalised to $100 \%$. Black bars, EGF treatment; white bars, no EGF treatment

production of NEUROG3 and in the latter stage increased the expression of pancreatic beta cell marker genes, including $P d x 1, N k x 6-1$ and Iapp (Fig. 5). Recently, Rolletschek et al. reported that our previous mouse protocol could lead ESCs into both pancreatic and neuronal differentiation [9]; however, we did not detect expression of neural marker beta-III tubulin in the C-peptide-positive cells at the final differentiation stage in our newly improved system (Fig. 4d).

Recently, several groups have reported protocols for the differentiation of insulin-positive cells from human ESCs $[4,6]$. However, insulin and glucagon double-positive cells were observed at the final differentiation stage. In pancreatic development, the mutually exclusive expression of endocrinal insulin and glucagon in the mature islet is crucial, because the doubly positive cells observed in the embryo pancreas have been shown by lineage tracing analysis to never give rise to adult endocrine cells as reviewed [10]. In our system, presence of glucagon in the C-peptide-positive clusters at the final differentiation stage was not detected (Fig. 3b), which suggested that the insulin-producing cells obtained by the present protocol were comparatively more mature.

Compared with freshly isolated mouse islets, the differentiated NT-ESCs revealed a quite lower level of insulin secretion $(0.53 \pm 0.04 \mathrm{vs} 9.3 \pm 3.2 \mathrm{pmol} / \mathrm{mg}$ at $2.5 \mathrm{mmol} / 1$ glucose; $1.15 \pm 0.26$ vs $39.3 \pm 5.4 \mathrm{pmol} / \mathrm{mg}$ at $27.5 \mathrm{mmol} / 1$ glucose). However, considering only 5 to $10 \%$ final differentiated cells were C-peptide-positive, our results were reasonable. In the future, we will establish a method to purify the beta cell from the differentiated cells and further evaluate the physiological function of ESC-derived beta cells.

In the therapeutic cloning approach, the low developmental potential of cloned embryos needs to be improved to yield a high efficiency for derived NT-ESCs. We obtained a high ratio of cloned cleavage embryos and successfully established four ESC lines from nine cloned blastocysts $(44.4 \%)$ in the present study. To acquire better NT efficiency, donor cells are generally subjected to a period of contact inhibition or serum starvation [11] for synchronisation at the G0/G1 phase. In our experiment, we combined the contact inhibition and serum starvation treatment to synchronise the donors and obtained improved cleavage of cloned embryos. It should also be noted that we found that insulin promoted proliferation of the ICM in cloned embryos, which is consistent with reported work on normal embryos [12].

In summary, the present study demonstrates that mouse NT-ESCs can differentiate into functional pancreatic beta cells and reverse diabetes in mice, illustrating the potential value of therapeutic cloning for type 1 diabetes mellitus cell therapy.

Acknowledgements This research was supported by Ministry of Science and Technology grant (2006AA02A113), Ministry of Education grant (705001), Science and Technology Plan of Beijing Municipal Government (D07050701350705), Gongjian project of Beijing Municipal Education Commission, National Basic Research Program for China (973 Program 2007CB947900), National Natural Science Foundation of China for Creative Research Groups (30421004) and a 111 Project to H. Deng. We thank L. Qian and F. Tang for a critical reading of the manuscript. We thank $\mathrm{D}$. Lu for the histological analysis of teratoma tissue, Z. Tong for the excellent technique of isolating mouse islets and Y. Zhang for technical support on real-time PCR. We also thank X. Sui, D. Zhao, X. Sun, M. Liu, P. Zhang, T. Qing, J. You, Y. Shen, W. Wei and other colleagues in our laboratory for technical assistance and advice during experiments.

Duality of interest The authors declare that there is no duality of interest associated with this manuscript. 


\section{References}

1. Rideout WM III, Hochedlinger K, Kyba M, Daley GQ, Jaenisch R (2002) Correction of a genetic defect by nuclear transplantation and combined cell and gene therapy. Cell 109:17-27

2. Soria B, Roche E, Berná G, León-Quinto T, Reig JA, Martín F (2000) Insulin-secreting cells derived from embryonic stem cells normalize glycemia in streptozotocin-induced diabetic mice. Diabetes 49:157-162

3. Shi Y, Hou L, Tang F et al (2005) Inducing embryonic stem cells to differentiate into pancreatic beta cells by a novel three-step approach with activin A and all-trans retinoic acid. Stem Cells 23:656-662

4. D'Amour KA, Bang AG, Eliazer S et al (2006) Production of pancreatic hormone-expressing endocrine cells from human embryonic stem cells. Nature Biotechnol 24:1392-1401

5. Jiang W, Shi Y, Zhao D et al (2007) In vitro derivation of functional insulin-producing cells from human embryonic stem cells. Cell Res 17:333-344
6. Jiang J, Au M, Lu K et al (2007) Generation of insulin-producing islet-like clusters from human embryonic stem cells. Stem Cells 25:1940-1953

7. Bai Z, Yong J, Qing T et al (2007) Serial nuclear transfer improves the developmental potential of mouse embryos cloned from oocytes matured in a protein-free medium. Mol Reprod Dev 74:560-567

8. Erbach GT, Lawitts JA, Papaioannou VE, Biggers JD (1994) Differential growth of the mouse preimplantation embryo in chemically defined media. Biol Reprod 50:1027-1033

9. Rolletschek A, Kania G, Wobus AM (2006) Generation of pancreatic insulin-producing cells from embryonic stem cells-'Proof of principle', but questions still unanswered. Diabetologia 49:2541-2545

10. Herrera PL, Nepote V, Delacour A (2002) Pancreatic cell lineage analyses in mice. Endocrine 19:267-278

11. Cibelli JB, Stice SL, Golueke PJ et al (1998) Cloned transgenic calves produced from nonquiescent fetal fibroblasts. Science 280:1256-1258

12. Lin TC, Yen JM, Gong KB, Hsu TT, Chen LR (2003) IGF-1/ IGFBP-1 increases blastocyst formation and total blastocyst cell number in mouse embryo culture and facilitates the establishment of a stem-cell line. BMC Cell Biol 4:14 